Open J. Math. Sci., Vol. 2(2018), No. 1, pp. 209 - 220

Website: https://pisrt.org/psr-press/journals/oms/

ISSN: 2523-0212 (Online) 2616-4906 (Print)

http://dx.doi.org/10.30538/oms2018.0029

\title{
FLIP AND HOPF BIFURCATIONS OF DISCRETE-TIME FITZHUGH-NAGUMO MODEL
}

\author{
QAMAR DIN ${ }^{1}$, SADAF KHALIQ
}

\begin{abstract}
In this paper, dynamics of a two-dimensional Fitzhugh-Nagumo model is discussed. The discrete-time model is obtained with the implementation of forward Euler's scheme. We present the parametric conditions for local asymptotic stability of steady-states. It is shown that the two-dimensional discrete-time model undergoes period-doubling bifurcation and Neimark-Sacker bifurcation at its positive steady-state. Furthermore, in order to illustrate theoretical discussion some interesting numerical examples are presented.
\end{abstract}

Mathematics Subject Classification: 39A30, 40A05, 92D25, 92C50.

Key words and phrases: Fitzhugh-Nagumo model; Flip bifurcation; Hopf bifurcation.

\section{Introduction}

In 1961 FitzHugh and Nagumo [1] presented the following two-dimensional model:

$$
\left(\begin{array}{c}
\frac{d x}{d t} \\
\frac{d y}{d t}
\end{array}\right)=\left(\begin{array}{c}
c_{1}\left(x+y-\frac{x^{3}}{3}\right) \\
\frac{1}{c_{1}}\left(x-a_{1}+b_{1} y\right)
\end{array}\right),
$$

where $a_{1}, b_{1}$ and $c_{1}$ are positive constants. Using the forward Euler method to system (1), we get the discrete-time model as follows:

$$
\left(\begin{array}{l}
x \\
y
\end{array}\right) \rightarrow\left(\begin{array}{c}
x+h c_{1}\left(x+y-\frac{x^{3}}{3}\right) \\
y-\frac{h}{c_{1}}\left(x-a_{1}+b_{1} y\right)
\end{array}\right)
$$

Received 24 February 2018. Revised 10 August 2018.

${ }^{1}$ Corresponding Author

(C) 2018 Qamar Din and Sadaf Khaliq. This is an open access article distributed under the Creative Commons Attribution License, which permits unrestricted use, distribution, and reproduction in any medium, provided the original work is properly cited. 
where $h>0$ is step size. For further biological relevance and dynamical analysis of some models that are very close to system (2), we refer to $[2,3,4,5,6$, $7,8,9,10,11,12,13,14,15]$, and references are therein. We investigate the existence of equilibria for (2) and local asymptotic stability of these steady-states by implementing linearized stability analysis techniques. Also, Neimark-Sacker bifurcation and period-doubling bifurcation are discussed.

\section{Existence of equilibria and stability}

The steady-states of (2) satisfy the following system of algebraic equations:

$$
x=x+h c_{1}\left(x+y-\frac{x^{3}}{3}\right), y=y-\frac{h}{c_{1}}\left(x-a_{1}+b_{1} * y\right) .
$$

From (3), it is quite simple to obtain:

$$
b_{1} x s+3\left(1-b_{1}\right) x-3 a_{1}=0, y=\frac{a_{1}-x}{b_{1}} .
$$

Next, we define the following quantity:

$$
\Delta:=4 b_{1}\left(b_{1}-1\right)^{3}-9 a_{1}^{2} b_{1}^{2} .
$$

Then, it follows that

- If $\Delta>0$, then system (2) has three distinct equilibrium points.

- If $\Delta=0$, then system (2) has a multiple equilibrium points.

- If $\Delta<0$, then system (2) has a unique positive equilibrium point.

For $a_{1}=4.92$ and $b_{1}=0.16$, we have $\Delta=-5.95649<0$ and existence for unique positive equilibrium is depicted in Figure 1. For $a_{1} \in[0,50]$ and $b_{1} \in[0,50]$, the region (blue) where $\Delta<0$ and region (red) where $\Delta>0$ are depicted in Figure 2. Mathematically, we have the following conditions for negativity and positivity of $\Delta$ :

- $\Delta<0$ if and only if $0<b_{1} \leq 1$, or $b_{1}>1$ and $a_{1}>\frac{2}{3} \sqrt{\frac{-1+3 b_{1}-3 b_{1}^{2}+b_{1}^{3}}{b_{1}}}$.

- $\Delta>0$ if and only if $b_{1}>1$ and $a_{1}<\frac{2}{3} \sqrt{\frac{-1+3 b_{1}-3 b_{1}^{2}+b_{1}^{3}}{b_{1}}}$.

- $\Delta=0$ if and only if $b_{1}>1$ and $a_{1}=\frac{2}{3} \sqrt{\frac{-1+3 b_{1}-3 b_{1}^{2}+b_{1}^{3}}{b_{1}}}$.

Now the Jacobian matrix of (2) evaluated at arbitrary equilibrium $(x, y)$ is given by:

$$
J(x, y):=\left(\begin{array}{cc}
1+\left(h-h x^{2}\right) c_{1} & h c_{1} \\
-\frac{h}{c_{1}} & 1-\frac{h b_{1}}{c_{1}}
\end{array}\right) .
$$

Moreover, the characteristic polynomial of $J(x, y)$ is given by:

$$
\begin{aligned}
P(\lambda) & :=\lambda^{2}-\left(2-\frac{b_{1} h}{c_{1}}+c_{1} h\left(1-x^{2}\right)\right) \lambda \\
& +1+h\left(c_{1}+h-c_{1} x^{2}\right)-\frac{b_{1} h\left(1+c_{1} h\left(1-x^{2}\right)\right)}{c_{1}} .
\end{aligned}
$$




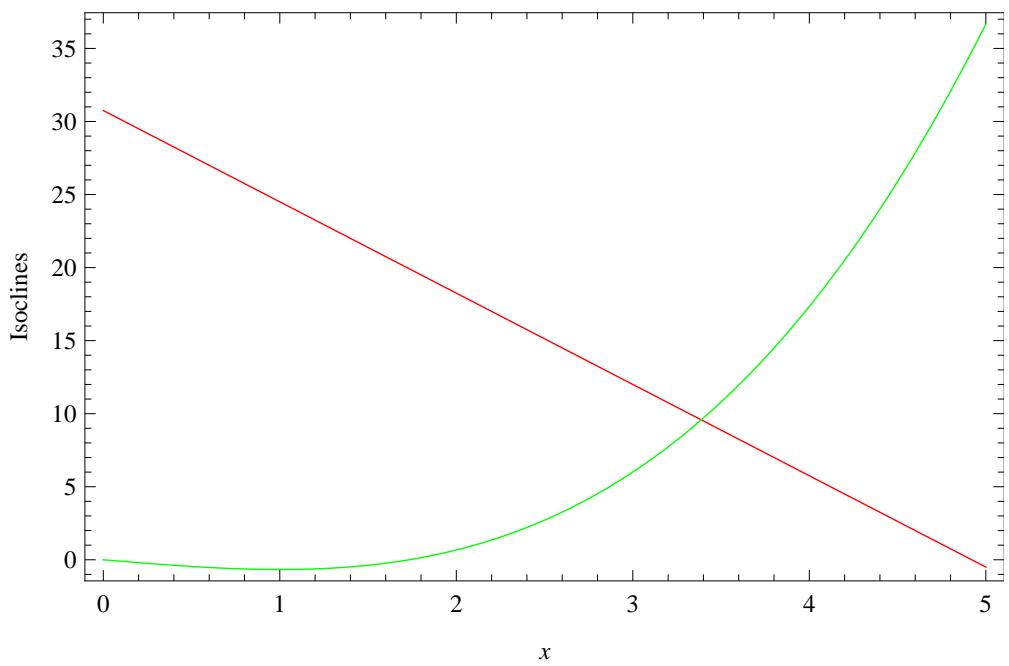

FiguRE 1. For $a_{1}=4.92$ and $b_{1}=0.16$ existence of unique positive equilibrium for $(2)$

Theorem 2.1. [16] Assume that $\Delta<0$, then unique positive equilibrium $(x, y)$ has the following topological classification:

(i) $(x, y)$ is a sink if and only if

$\left|2-\frac{b_{1} h}{c_{1}}+c_{1} h\left(1-x^{2}\right)\right|<2+h\left(c_{1}+h-c_{1} x^{2}\right)-\frac{b_{1} h\left(1+c_{1} h\left(1-x^{2}\right)\right)}{c_{1}}<2$.

(ii) $(x, y)$ is a saddle if and only if

$\left(2-\frac{b_{1} h}{c_{1}}+c_{1} h\left(1-x^{2}\right)\right)^{2}-4\left(1+h\left(c_{1}+h-c_{1} x^{2}\right)-\frac{b_{1} h\left(1+c_{1} h\left(1-x^{2}\right)\right)}{c_{1}}\right)>0$,

and

$\left|2-\frac{b_{1} h}{c_{1}}+c_{1} h\left(1-x^{2}\right)\right|>\left|2+h\left(c_{1}+h-c_{1} x^{2}\right)-\frac{b_{1} h\left(1+c_{1} h\left(1-x^{2}\right)\right)}{c_{1}}\right|$.

(iii) $(x, y)$ is a source if and only if

$$
\left|1+h\left(c_{1}+h-c_{1} x^{2}\right)-\frac{b_{1} h\left(1+c_{1} h\left(1-x^{2}\right)\right)}{c_{1}}\right|>1,
$$

and

$$
\left|2-\frac{b_{1} h}{c_{1}}+c_{1} h\left(1-x^{2}\right)\right|<\left|2+h\left(c_{1}+h-c_{1} x^{2}\right)-\frac{b_{1} h\left(1+c_{1} h\left(1-x^{2}\right)\right)}{c_{1}}\right| .
$$




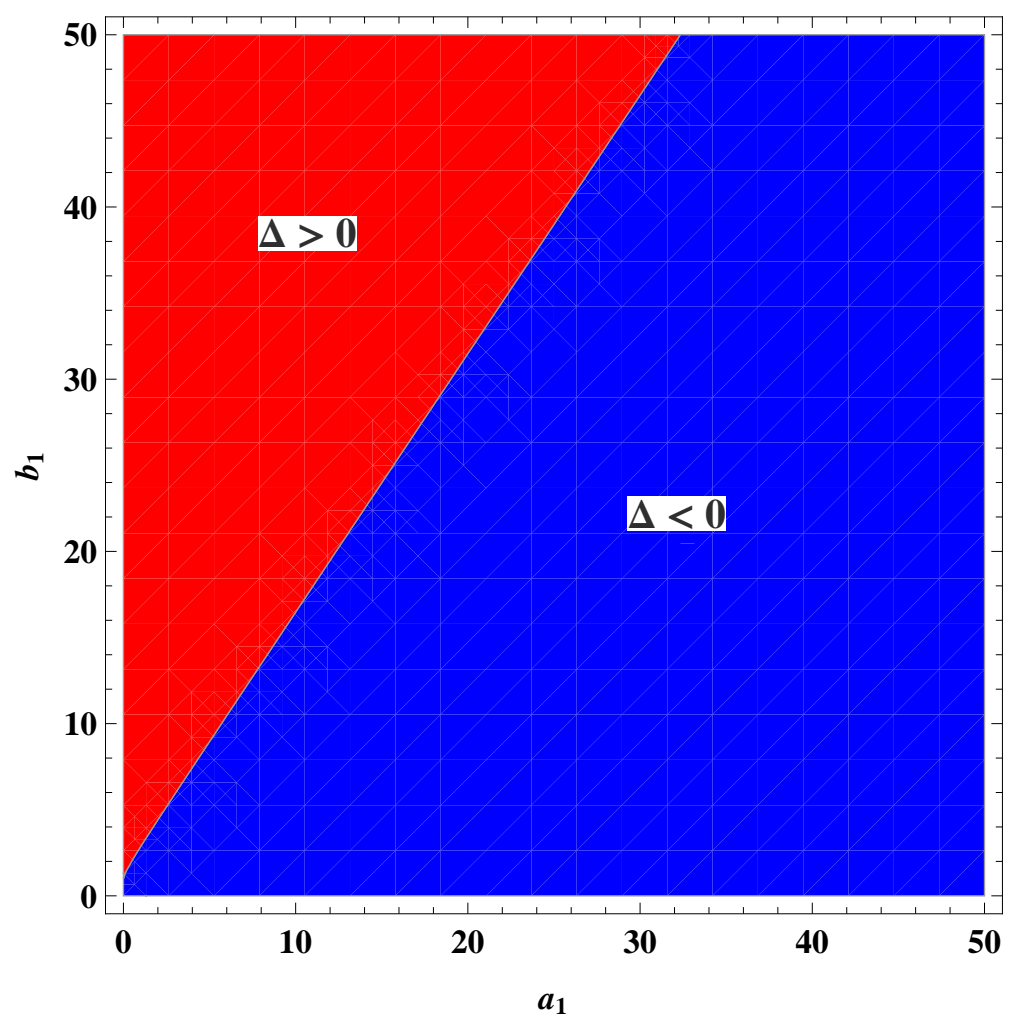

FiguRE 2. Regions for existence of various equilibria

(iv) $(x, y)$ is non-hyperbolic if and only if

$$
\left|2-\frac{b_{1} h}{c_{1}}+c_{1} h\left(1-x^{2}\right)\right|=\left|2+h\left(c_{1}+h-c_{1} x^{2}\right)-\frac{b_{1} h\left(1+c_{1} h\left(1-x^{2}\right)\right)}{c_{1}}\right|,
$$

or

$$
\left(c_{1}+h-c_{1} x^{2}\right)-\frac{b_{1}\left(1+c_{1} h\left(1-x^{2}\right)\right)}{c_{1}}=0
$$

and

$$
\left|2-\frac{b_{1} h}{c_{1}}+c_{1} h\left(1-x^{2}\right)\right| \leq 2 .
$$

If we choose $b_{1}=0.45, c_{1}=0.15, h \in[0,1]$ and $x \in[0,10]$, then topological classification for unique positive point of system (2) is shown in Figure 3. 


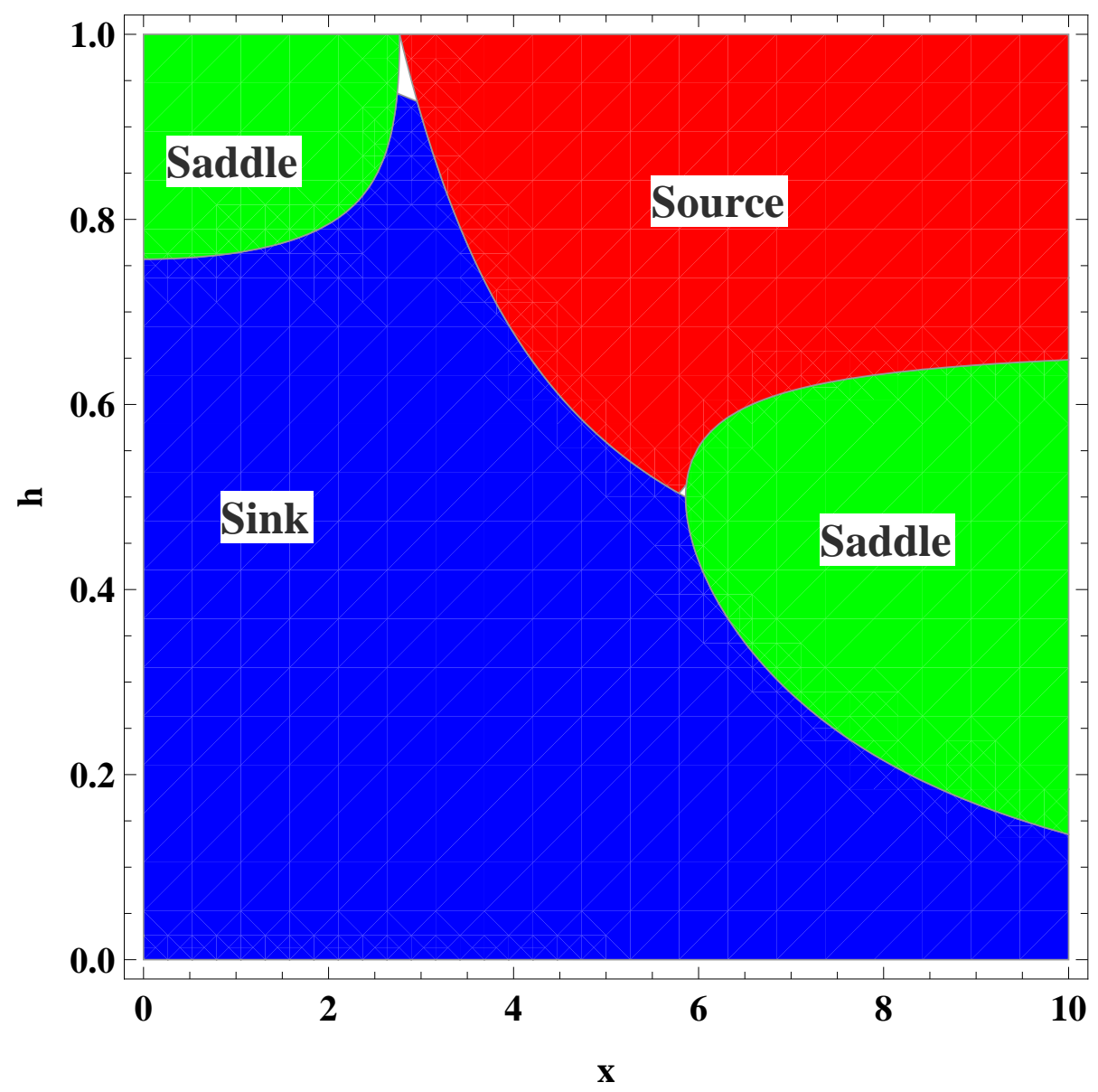

Figure 3. Topological classification for positive equilibrium

\section{Bifurcation analysis}

Studying bifurcation analysis for discrete-time models is a topic of great interest. Recently, there are many articles have published for the investigation for perioddoubling and Neimark-Sacker bifurcations in discrete-time models [17, 18, 19, 20, $21,22,23]$. In this section, we explore the parametric conditions under which system (2) undergoes period-doubling and Neimark-Sacker bifurcations at its unique positive equilibrium point. For this, first we discuss the emergence of period-doubling bifurcation at positive equilibrium of system (2). Assume that 
$P(-1)=0$, where $P(\lambda)$ is defined in (5), then system (2) undergoes perioddoubling bifurcation as $h$ varies in a small neighborhood of $h_{0}$ defined by

$$
h_{0}:=\frac{b_{1}+\left(-1+x^{2}\right) c_{1}^{2}-\sqrt{-4 c_{1}^{2}+\left(b_{1}-\left(-1+x^{2}\right) c_{1}^{2}\right)^{2}}}{\left(1+\left(-1+x^{2}\right) b_{1}\right) c_{1}},
$$

or

$$
h_{0}:=\frac{b_{1}+\left(-1+x^{2}\right) c_{1}^{2}+\sqrt{-4 c_{1}^{2}+\left(b_{1}-\left(-1+x^{2}\right) c_{1}^{2}\right)^{2}}}{\left(1+\left(-1+x^{2}\right) b_{1}\right) c_{1}} .
$$

Secondly, we assume that

$$
\left(b_{1}+\left(-1+x^{2}\right) c_{1}^{2}\right)^{2}\left(-4 c_{1}^{2}+\left(b_{1}-\left(-1+x^{2}\right) c_{1}^{2}\right)^{2}\right)<0 .
$$

Then system (2) undergoes Neimark-Sacker bifurcation as parameter $h$ varies in a small neighborhood of $h_{1}$ defined by:

$$
h_{1}:=\frac{b_{1}+\left(-1+x^{2}\right) c_{1}^{2}}{\left(1+\left(-1+x^{2}\right) b_{1}\right) c_{1}} .
$$

In order to verify aforementioned mathematical investigation for existence of period-doubling and Neimark-Sacker bifurcations, we choose particular parametric values for system (2) as follows:

Period-doubling bifurcation: Let $a_{1}=2.6, b_{1}=1.2, c_{1}=1.9$ and $h \in[0.3,0.5]$. In this case, system (2) undergoes period-doubling bifurcation as $h$ varies in a small neighborhood of $h_{0}=0.39$. Moreover, the bifurcation diagrams for period-doubling bifurcation are shown in Figure 4 and Figure 5. Moreover, maximum Lyapunov exponents (MLE) are shown in Figure 6 and a chaotic attractor is depicted in Figure 7.

Neimark-Sacker bifurcation: Taking $a_{1}=2.7, b_{1}=2.5, c_{1}=0.95$ and $h \in[0.65,0.72]$. Then system (2) undergoes Neimark-Sacker bifurcation as $h$ varies in a small neighborhood of $h_{1}=0.69$. The diagrams for Neimark-Sacker bifurcation are given in Figure 8 and Figure 9. Furthermore, MLE are shown in Figure 10 and phase portrait at $h=0.69$ is depicted in Figure 11.

\section{Conclusion}

The qualitative behavior for a two-dimensional discrete-time Fitzhugh-Nagumo model is investigated. Euler's forward scheme is implemented to obtain the discrete counterpart of the continuous Fitzhugh-Nagumo model. It is investigated that discrete-time model has rich dynamical behavior as compare to its continuous counterpart. The topological classification for steady-state solutions is discussed. Furthermore, parametric conditions for the existence of perioddoubling bifurcation and Neimark-Sacker bifurcation are analyzed by taking $h$ as bifurcation parameter. At the end numerical simulations are provided to illustrate the theoretical discussion. 


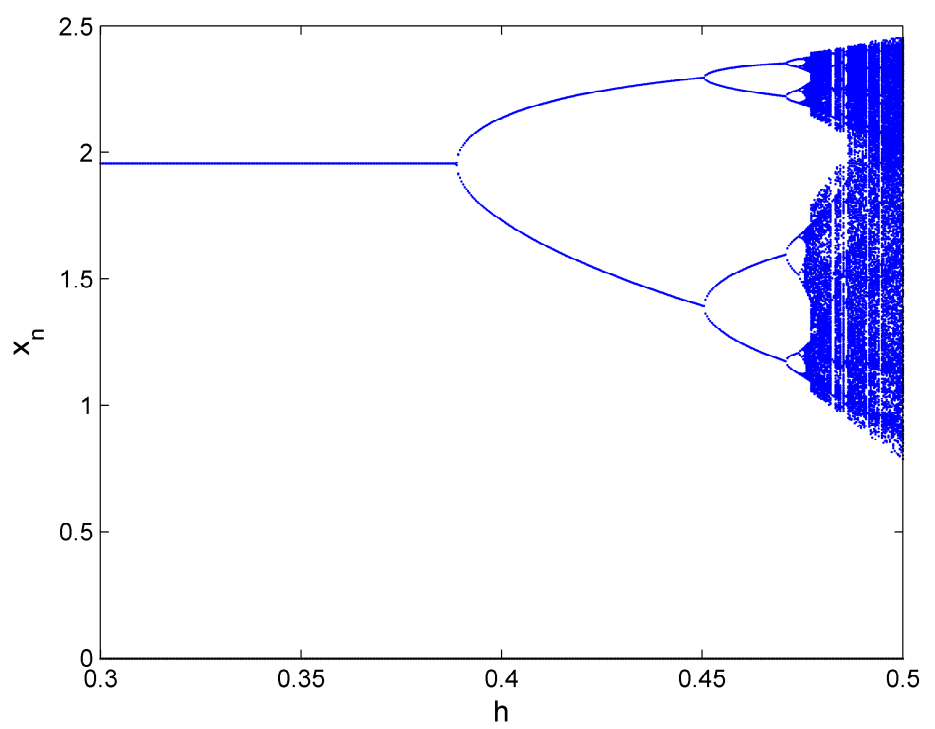

FIGURE 4. Bifurcation diagram for $x_{n}$

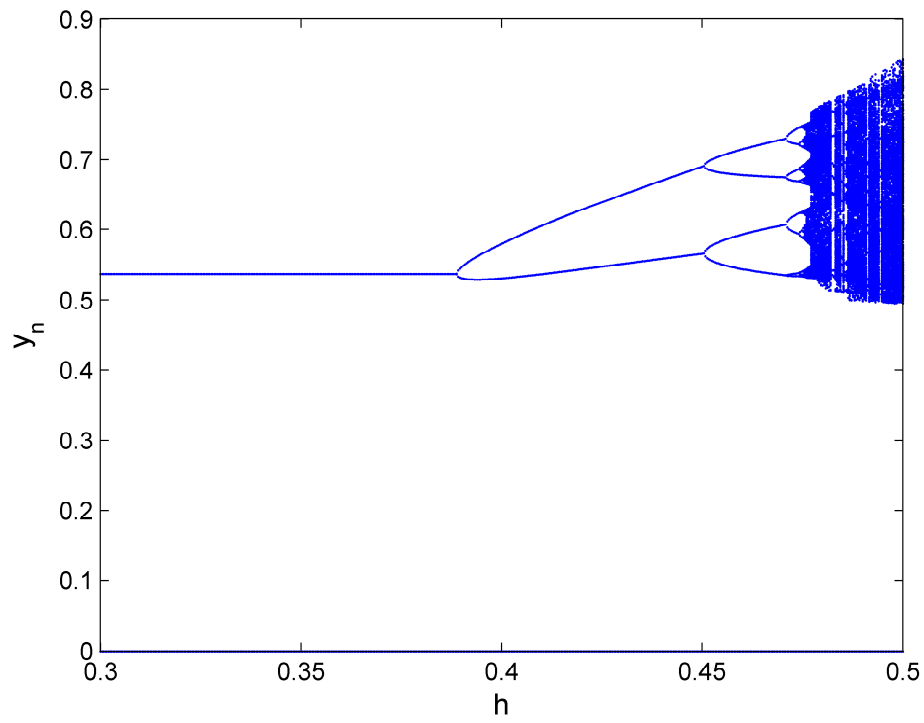

FIGURE 5. Bifurcation diagram for $y_{n}$ 


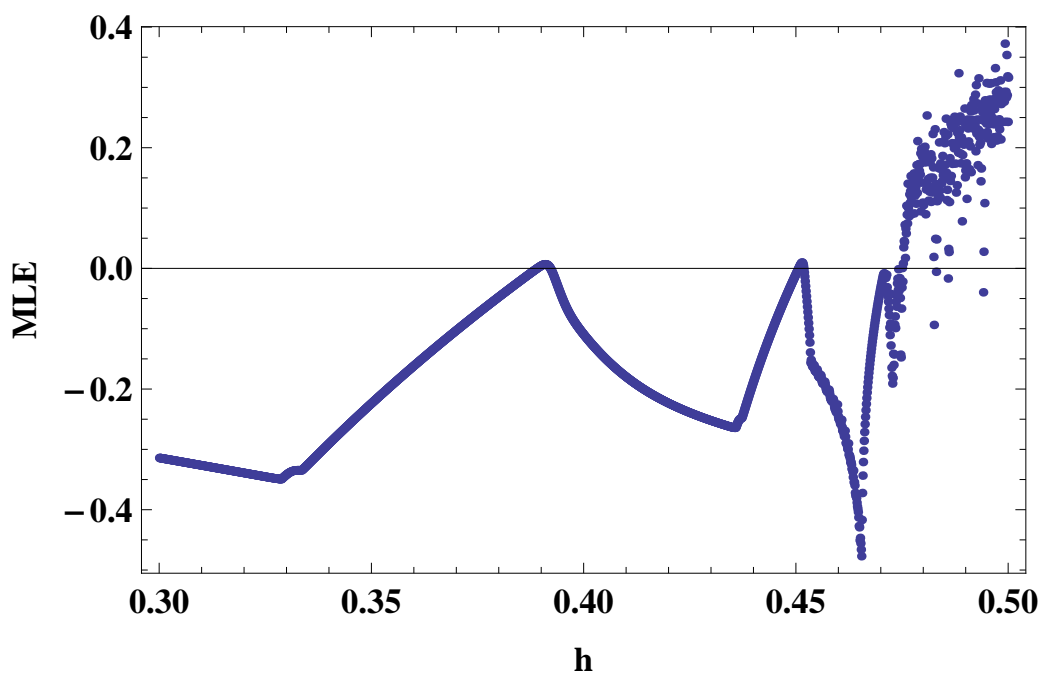

Figure 6. Maximum Lyapunov exponents

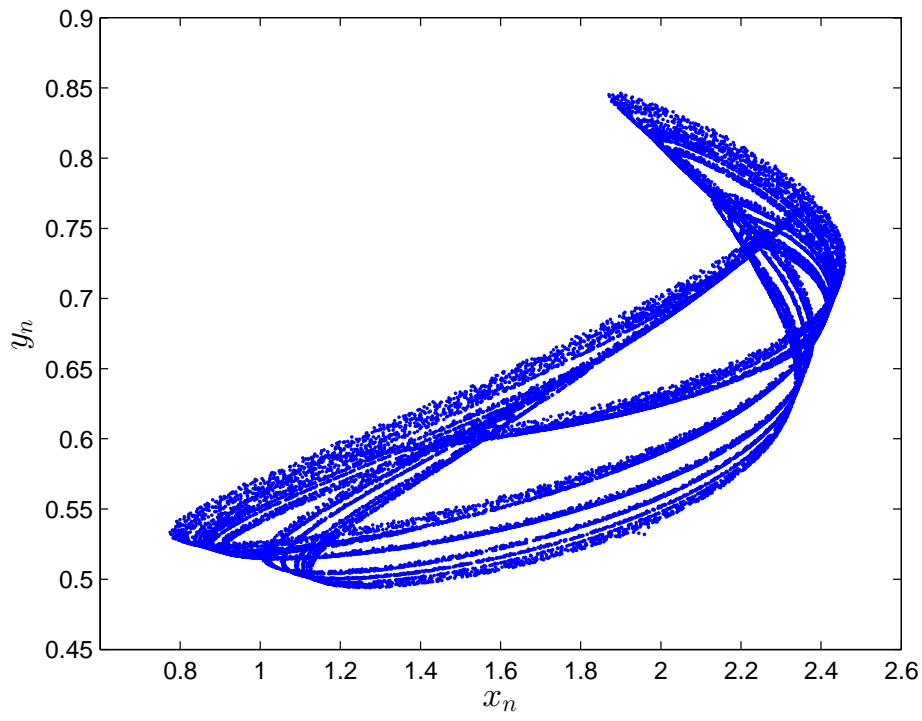

FiguRe 7. A chaotic attractor at $h=0.5$ 


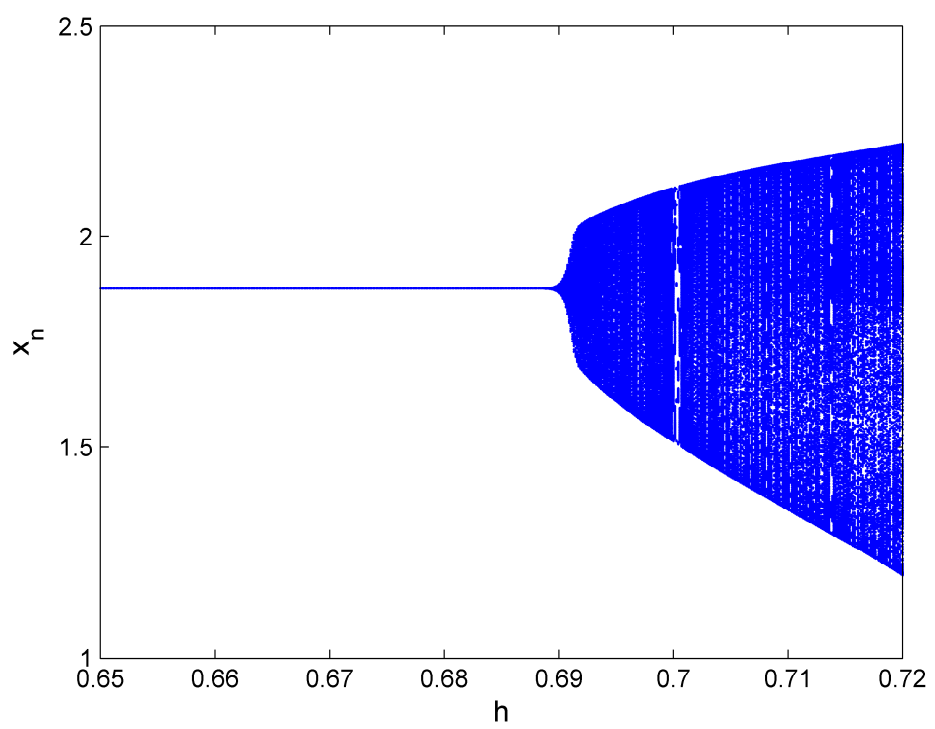

Figure 8. Bifurcation diagram for $x_{n}$

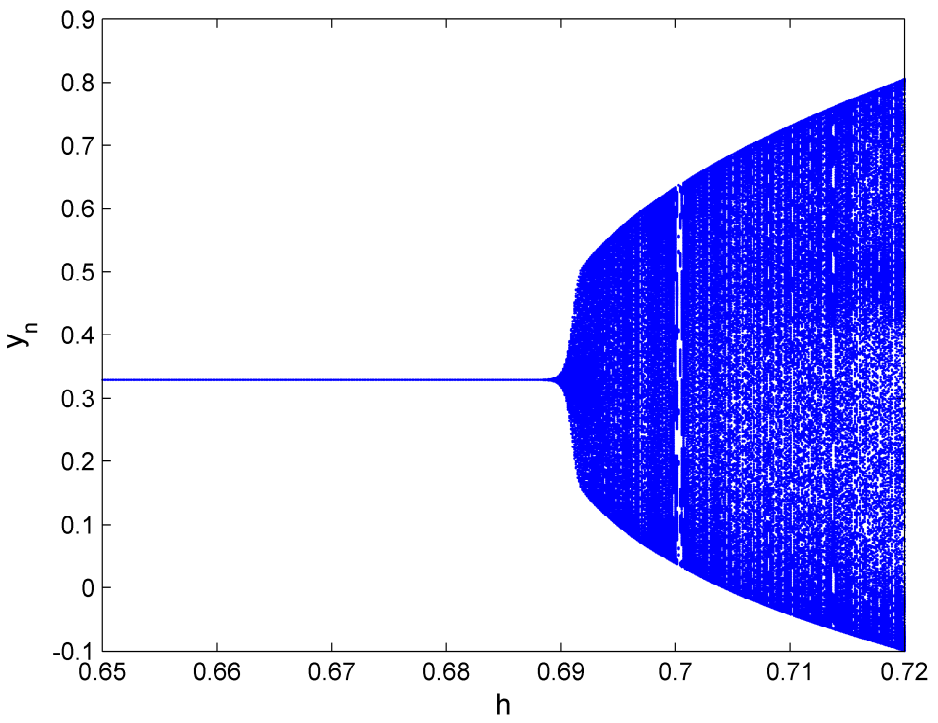

Figure 9. Bifurcation diagram for $y_{n}$ 


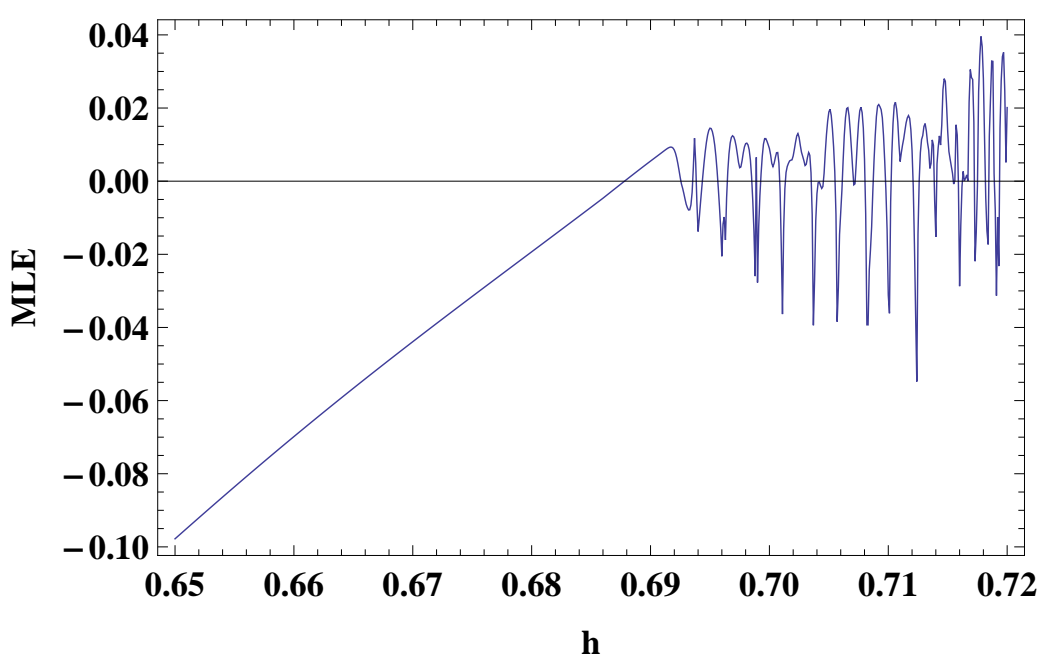

Figure 10. Maximum Lyapunov exponents

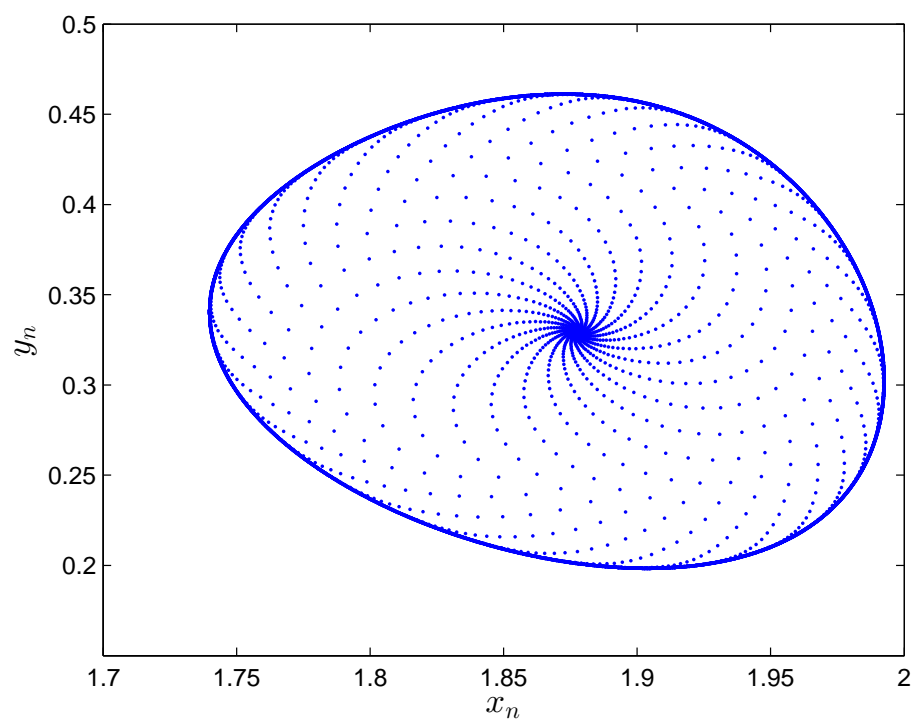

Figure 11. Phase portrait at $h=0.69$ 


\section{Competing Interests}

The author(s) do not have any competing interests in the manuscript.

\section{REFERENCES}

1. FitzHugh, R. (1961). Impulses and physiological state in theoretical models of nerve membrane. Biophysical Journal, 1(6), 445-467.

2. Hodgkin, A. L., \& Huxley, A. F. (1952). A quantitive description of membrane current and its application to conduction and excitation in nerve. The Journal of Physiology, 117(4), 500-544.

3. Hindmarsh, J. L., \& Rose, R. M. (1984). A model of neuronal bursting using three coupled first order differential equations. Biological Sciences, Proceedings of the Royal Society of London B, 221(1222), 87-102. http://dx.doi.org/10.1098/rspb.1984.0024

4. Buzzi, C., Llibre, J., \& Medrado, J. (2015). Hopf and zero-Hopf bifurcations in the Hindmarsh-Rose system. Nonlinear Dynamics, 83(3), 1549-1556. https://doi.org/10.1007/s11071-015-2429-y

5. Chay, T. R., \& Keizer, J. (1985). Theory of the effect of extracellular potassium on oscillations in the pancreatic beta cell. Biophysical Journal, 48(5), 815-827. https://doi.org/10.1016/S0006-3495(85)83840-6

6. Chay, T. R., \& Rinzel, J. (1985). Bursting, beating and chaos in an excitable membrane model. Biophysical Journal, 47(3) 357-366. https://doi.org/10.1016/S0006-3495(85)839266

7. Chay, T. R. (1985). Chaos in a three-variable model of an excitable cell. Physical Dynamics, 16(2), 233-242. https://doi.org/10.1016/0167-2789(85)90060-0

8. Chay, T. R. (1985). Glucose response to bursting-spiking pancreatic beta-cells by a barrier kinetic model. Biological Cybernetics, 52(5), 339-349.

9. Chen, S. S., Cheng, C. Y., \& Rulin, Y. (2013). Application of a two-dimensional Hindmarsh-Rose type model for bifurcation analysis. International Journal of Bifurcation and Chaos, 23(3), 1350055. https://doi.org/10.1142/S0218127413500557

10. Innocenti, G., Morelli, A., Genesio, R., \& Torcini, A. (2007). Dynamical phases of the Hindmarsh-Rose neuronal model: Studies of the transition from bursting to spiking chaos. Chaos, 17(4), 043128. https://doi.org/10.1063/1.2818153

11. Jing, Z. J., Chang, Y., \& Guo, B. L. (2004). Bifurcation and chaos in discrete FitzHugh-Nagumo system. Chaos, Solitons and Fractals, 21(3), 701-720. https://doi.org/10.1016/j.chaos.2003.12.043

12. Liu, X. L., \& Liu, S. Q. (2012). Codimension-two bifurcation analysis in two dimensional Hindmarsh-Rose model. Nonlinear Dynamics, 67(1), 847-857. https://doi.org/10.1007/s11071-011-0030-6

13. Lange, E., \& Hasler, M. (2008). Predicting single spikes and spike patterns with the Hindmarsh-Rose model. Biological Cybernetics, 99: 349. https://doi.org/10.1007/s00422008-0260-y

14. Li, B., \& He, Z. (2014). Bifurcation and chaos in a two-dimensional discrete HindmarshRose model. Nonlinear Dynamics, 76(1), 697-715. https://doi.org/10.1007/s11071-0131161-8

15. Rocsoreanu, C., Georgescu, A., \& Giurgiteanu, N. (2000). FitzHugh-Nagumo model: Bifurcation and Dynamics. Springer, Dordrechts. ISBN 978-94-015-9548-3

16. Kulenović, M. R. S., \& Ladas, G. (2002). Dynamics of Second Order Rational Difference Equations: With Open Problems and Conjectures. Chapman and Hall/CRC, New York. ISBN 9781584882756

17. Din, Q. (2018). A novel chaos control strategy for discrete-time Brusselator models. Journal of Mathematical Chemistry. https://doi.org/10.1007/s10910-018-0931-4 
18. Din, Q., \& Hussain, M. (2018). Controlling chaos and Neimark-Sacker bifurcation in a host-parasitoid model. Asian Journal of Control. https://doi.org/10.1002/asjc.1809

19. Din, Q., Elsadany, A. A., \& Ibrahim, S. (2018). Bifurcation analysis and chaos control in a second-order rational difference equation. International Journal of Nonlinear Sciences and Numerical Simulation, 19(1), 53-68. https://doi.org/10.1515/ijnsns-2017-0077

20. Din, Q. (2018). Bifurcation analysis and chaos control in discrete-time glycolysis models. Journal of Mathematical Chemistry. 56(3): 904-931. https://doi.org/10.1007/s10910-017$0839-4$

21. Din, Q., Donchev, T., \& Kolev, D. (2018). Stability, Bifurcation Analysis and Chaos Control in Chlorine Dioxide-Iodine-Malonic Acid Reaction. MATCHCommunications in Mathematical and in Computer Chemistry, 79(3), 577-606. https://doi.org/10.1007/s10910-017-0839-4

22. Din, Q. (2018). Controlling chaos in a discrete-time prey-predator model with Allee effects. International Journal of Dynamics and Control, 6(2), 858-872. https://doi.org/10.1007/s40435-017-0347-1

23. Din, Q. (2018). Qualitative analysis and chaos control in a density-dependent hostparasitoid system. International Journal of Dynamics and Control, 6(2), 778-798. https://doi.org/10.1007/s40435-017-0341-7

\section{Qamar Din}

Department of Mathematics, The University of Poonch Rawalakot, Pakistan.

e-mail: qamar.sms@gmail.com

Sadaf Khaliq

Department of Mathematics, The University of Poonch Rawalakot, Pakistan. e-mail: sadafkhaliq00@gmail.com 